\title{
Systematic review of the evidence for sustained efficacy of dietary interventions for reducing appetite or energy intake
}

\author{
Jason C. G. Halford ${ }^{1}$, Una Masic ${ }^{1}$, Cyril F. M. Marsaux ${ }^{2}$, Andrew J. Jones ${ }^{1}$, Anne Lluch ${ }^{3}$, \\ Luca Marciani ${ }^{4}$, Monica Mars ${ }^{5}$, Sophie Vinoy ${ }^{6}$, Margriet Westerterp-Plantenga ${ }^{7}$ and \\ David J. Mela ${ }^{8}$ \\ ${ }^{1}$ Institute of Psychology, Health and Society, University of Liverpool, Liverpool, United Kingdom, \\ ${ }^{2}$ ILSI Europe, Brussels, Belgium, \\ ${ }^{3}$ Centre Daniel Carasso, Danone Nutricia Research, Palaiseau, France, \\ ${ }^{4}$ NIHR Nottingham Biomedical Research Centre at Nottingham University Hospitals NHS Trust and the University of \\ Nottingham, Nottingham, United Kingdom, \\ ${ }^{5}$ Division of Human Nutrition, Wageningen University and Research, Wageningen, Netherlands, \\ ${ }^{6} R \& D$, Nutrition Department, Mondelèz International, Clamart, France, \\ ${ }^{7}$ NUTRIM School of Nutrition and Translational Research in Metabolism, Maastricht University, Maastricht, \\ Netherlands and \\ ${ }^{8}$ Formerly with Unilever R\&D Vlaardingen, Vlaardingen, Netherlands
}

\begin{abstract}
Introduction: In the broad literature on the effects of ingredients, foods and diets on appetite and energy intake (EI), most trials involve a single acute intervention. It is unclear whether these acute results are generally sustained over longer periods. Researchers and regulators therefore lack an objective basis to judge the appropriate duration of efficacy trials in appetite control, to have confidence that acute effects are likely to be maintained. This gap creates uncertainty in requirements and study designs for the substantiation of satiety-enhancing approaches to help in controlling eating behaviour.
\end{abstract}

Materials and Methods: A systematic search of literature (Prospero registration number CRD42015023686) identified studies testing both the acute and chronic effects of food-based interventions aimed at reducing appetite or EI. From 9680 unique records identified from titles and abstracts, 178 papers were selected for full screening. Twenty-six trials met the inclusion criteria and provided data sufficient for use in this analysis, and were also scored for risk of bias (RoB) indicators.

Results: Most of these trials (21/26) measured appetite outcomes and over half (14/26) had objective measures of EI. A significant acute effect of the intervention was retained in 10 of 12 trials for appetite outcomes, and six of nine studies for EI. Initial effects were most likely retained where these were more robust and studies adequately powered. Where the initial, acute effect was not statistically significant, a significant effect was later observed in only two of nine studies for appetite and none of five studies for EI. The main sources of RoB were lack of a priori power calculations and failure to report analyses based on intention-to-treat. Furthermore, $12 / 26$ studies were not adequately powered to detect a meaningful reduction in appetite $(\sim 10 \%)$.

Discussion: Maintenance of acute intervention effects on appetite or EI need to be confirmed, but seems likely where the initially observed effects are robust and replicable in adequately powered studies.

Conflict of Interest: This work was conducted by an expert group of the European branch of the International Life Sciences Institute, ILSI Europe and coordinated by the Eating Behaviour and Energy Balance Task Force. Industry members of this task force are listed on ILSI Europe's website at http://ilsi.eu/task-forces/nutrition/eating-behaviour-and-energy- balance/.

\section{Reference}

1. Halford JCG, Masic U, Marsaux CFM, Jones AJ, Lluch A, Marciani L, Mars M, Vinoy S, Westerterp-Plantenga M, Mela DJ (2018) Systematic review of the evidence for sustained efficacy of dietary interventions for reducing appetite or energy intake. Obesity Reviews 19, $1329-1339$. 